\title{
The phenomenon of "Image Defilation" of Tourist Destination-Study on the Problem of Tourism Development in Weixi County
}

\author{
Lijun $\mathrm{Xu}^{1, \mathrm{a}}$ \\ ${ }^{1}$ Southwest Minzu University, Chengdu, Sichuan, 610000 \\ a'E-mail:xulijun316@126.com
}

Keywords: Image Defilation, Development Path of Tourism, Weixi County

\begin{abstract}
Weixi County is the largest population county of Diqing, Yunnan province and at the same time one of counties with the largest poverty population. It has the characteristics of combining with frontier, minority nationality and poverty. Developing tourism has become the largest prospect of overcoming poverty and achieving prosperity for the locals. There are two reasons for choosing Weixi County as the case location. The first reason is the urgency of Weixi County's tourism development. The second reason is that the surrounding tourist cities (such as Shangri-la, Lijiang, etc.) Have typical shadowing effects. Under the circumstances, how to overcome the shadowing effect, position reasonably the tourism destination image and look for development path have certain discussion values and practical significance. It can be seen that the image positioning of Weixi county shall follow the strategy of "forming differences under theme image" to explore special tourism resources and cultural resources in the county, to try to break the barrier of the respective area under the general background of brand Shangri-la ecological tourism zone, to introduce the concept of "Non-zero-sum cooperative game", to sake fellowship with surrounding tourism destinations and cooperative development of regional tourism circle.
\end{abstract}

\section{Introduction}

The competition among tourist destinations is to a large extent an image of competition. [1] This article takes Weixi Lisu Autonomous County as a case, and analyzes the problems existing in local tourism development and image positioning, and finds that it is in relation to the surrounding tourist cities. (Shangri-La City, Lijiang City, etc.) Have a typical shadowing effect. This article has carried on the thorough discussion and research on overcoming the shadowing effect, locating the tourism destination image reasonably, and seeking the development path of tourism.

\section{Literature Review}

Western tourism research focuses on "seeking" in the relationship between supply and demand. Through psychology research methods, Michael Garnner thinks that the image drives tourists to go for tourism. In 1979, Breton used geography to explore geographical images and realistic images. Impact on tourists. After the 1980s, it gradually formed a research shift from "pursuing" to "supply", that is, exploring the image orientation of tourism destinations. The tourism site image has the nature of marketing, which is the so-called "inducibility", which is the most attractive feature of tourist sites. Wang Lei et al. (1999) based on Kotler's concept of emissive image and receptive image, concluded that the conceptual system of the tourism destination image contains two aspects, ie, the emissive destination image and the receptive destination image. The intention is to visualize the establishment of the destination image as a systematic process that supports the overall marketing concept of the destination. [2] At the same time, tourism image design is the focus of tourism image research. Li Leilei (1998) believes that the core of urban tourism image design should be to solve the basic positioning problem of urban tourism. The further development of urban tourism cannot rely solely on isolation. Tourist attractions, but must introduce the city's overall tourism image, through the positioning of tourism image, the theme of the slogan, visual 
image design and promotion of basic image strategy to fully develop urban tourism. [3] Wu Bihu and Song Zhiqing (2000) established the basic procedures for designing travel images, including basic research in the early stage and display research in the latter stage. Image orientation is to put forward the overall image of regional tourism development and put forward the core content of tourism image, namely the overall image. [4]

With respect to the theoretical exploration of the image of China's tourism destinations, Wang Yanyong (1999)[5] suggested that tourism destinations with relatively low resource levels are vulnerable to the negative impact brought by higher-level tourism destinations and proposes "shadow areas". Theory, and pointed out that the theme image is difficult to cover all resources and should fully design the tourism image. Li Guoping and Ye Wen (2002) proposed a "grayscale area" and "aura area" that tourists perceive based on their perception of tourist images. It is pointed out that due to the mutuality and limitedness of the tourist source and the mutual competition between the tourist destinations, the tourism image of the "Halo District" has a huge shielding effect on the "grayscale area". [6] Yang Zhenzhi and Chen Jin (2003) proposed for the first time the concept of "image obscuration" and "image superposition" as the core theory of image planning, and conducted theoretical analysis and empirical research. It is concluded that the relationship between different tourism destinations is divided into two main types: competition (causing image obscuration) and image integration (leading to image superposition). [7] Liu Ruiwen (2006) proposed the tourism image asymmetry role theory emphasizes the differences in the image levels of different tourism destinations, leading to the asymmetry in the image, as well as changes in tourism behavior and tourism regional image. Its theoretical system framework includes the shadow region of tourism image theory, the shadow effect theory of tourism image, the gray region theory of tourism image and the time advantage theory of tourism image. [8] The proposed theory has great inspiration and reference significance for the theoretical and practical research on related issues of future tourism images.

In recent years, tourism research has also focused on the differences in the image of tourist destinations, the perception of tourism destinations on the level of tourists, and the study on the tourism image of newer and more competitive tourism districts. Ma Xiaojing (2006) believes that tourism image is a manifestation of tourism viewing mode. The viewing mode is influenced by world outlook, values, attitudes, tendencies, beliefs and other factors, and is the result of social and cultural influence. Therefore, the shaping of the tourism image is an extensive social and cultural process. [9] Wang Yuan et al. (2014) believe that the image of tourist destinations is changing dynamically, and time is an important dimension of the dynamic changes of tourism destinations. It also analyzes the evolution process of the perception image and the launch image of both the supplier and the tourists. Guo Fenghua (2015) thought that tourists' spontaneous uploading of travel notes in blogs had an important influence on the shaping and dissemination of the "Five Golden Flowers" tourism image of Chengdu Farmhouse. The article carefully studied the image of tourism destinations with different types of feature word groups. The study of rural tourism development provides new perspectives and tools. Zhu Xiangbo (2016) proposed a stereotype of tourists' perceptions of tourism as an important internal factor in the shadowing of tourist attractions in Panzhihua City through the study of the factors that explain the formation of tourist destinations. [10]

In researching the development of tourism in ethnic minority areas in China and related issues, Wu Bihu and Yu Qing (2000) obtained a statistical analysis of the literature on the development of Chinese ethnic culture tourism since 1985 and concluded that ethnic cultural tourism is one of the main trends of modern tourism. Raise awareness of the development of ethnic cultural tourism resources; conduct an all-round re-understanding of national culture, in-depth study of its connotations, and promote national cultural innovation; the development of ethnic cultural tourism resources is a gradual development process, and must be planned and staged in an integrated manner. Implementation; should use various media and means, especially public media and computer network means, increase publicity, establish a national cultural tourism image. [11] Zhang Guohong (2001) proposed that the development of cultural tourism products should be 
characterized by distinct cultural forms, rich tastes for cultural connotations, and humanistic spirits for the cultural essence; the concept of cultural tourism marketing requires market demand Through the constant innovation of cultural tourism products and services and marketable solutions, the organization and management of business decision-making will ultimately satisfy the needs of tourists. [12] Liu Xiaoying and Wu Yusheng (2004) analyzed the shortcomings of the specific implementation process in the strategy for the development of the western region, and put forward the development strategy of ecological construction and urbanization as well as the development of tourism in Sichuan ethnic areas. [13]

In recent years, more and more scholars have begun to study the economic, social, and ecological issues in the development of tourism in ethnic villages, including the problem of the retreat of national culture, the benefit of farmers participating in the project, and the tourism environment in the village. Wang Yuhui and Liu Wang (2009) took Taoping Qiang Village in Li County of Sichuan as an example to analyze the endogenous predicament and deep-rooted roots in the local tourism development through the intricacies of the particularity of the resource system and proposed a governance path. select. Wang Jun and Zhu Xiaohui (2012) proposed the importance of enterprises to the development model of the main tourism, and repositioned the government's role in the development of the Xishuangbanna Dai Park. Zhang Ji and Xu Hongyi (2016) through the phenomenon of conflict between host and guest roles in ethnic tourism, taking Baisha Village in Lijiang as a case, and based on the "space-power" theory, focused on the interaction of the role of the host and the visitor in the home space of the national tourism region. Conflict phenomenon. [14]

\section{Case Tourism Resources and Development Status}

Weixi County is the county with the largest population and the largest number of poor people in Diqing Prefecture, Yunnan Province. It has the characteristics of integrating border, ethnicity and poverty. The development of tourism has become the biggest vision for the local people to get rid of poverty. The case of Weixi County was selected as the case of this article. First, the urgency of developing tourism, and the second, the surrounding tourism counties and cities (Shangri-La City, Lijiang City, etc.) had a typical shadowing effect. Under this kind of objective reality, how to overcome the shadowing effect and find the development path has certain value and practical significance.

Weixi Lisu Autonomous County is located between latitude $26^{\circ} 53^{\prime} \sim 28^{\circ} 02^{\prime} \mathrm{N}$ and longitude 98 $54^{\prime} \sim 99^{\circ} 34^{\prime} \mathrm{E}$. It is located in the heart of the natural heritage of the Three Parallel Rivers of the Jinsha River, Lancang River and Nujiang River. The important part of the "Shangri-La" Wonderland is the only She Autonomous County in the country. The county is an excessive belt on the Qinghai-Tibet Plateau toward the Yunnan-Guizhou Plateau, adjacent to Shangri-La in the east, bordered by Lanping County in Nujiang Prefecture in the south, connected to Yulong County in Lijiang City in the south, and bordered by Gongshan and Fugong in the Nujiang Prefecture in the west, and on the northeast and back. It is adjacent to Deqin County. Weixi Lisu Autonomous County, with its unique geographical location, has become a hub from which Tibet enters Tibet and then to Southeast Asian countries, as well as a hub connecting Tibet and the mainland. Weixi County is known as the "green gems in Hengduan Mountain" and "Sanjiang Pearl". It is known as the "Congery Site of Chinese Lisu Culture," "Hometown of Medicinal Materials," "Hometown of Chinese Orchids," and "Plant Kingdom." "The animal kingdom." [15]

According to the distribution of Weixi tourism resources area, it is mainly divided into a center and two landscape corridors: Baohe Town Center Area, Shangri-La Golden Monkey National Park and Lancang River Landscape Corridor. The area of Baohe Town Center features natural rhododendron gardens, caves, and lyrics; Shangri-La's Golden Monkey National Park features religious attractions such as the Damo cave, wild animal rescue station, "Hamba" culture, and Mary Masa culture. Etc.; Lancang landscape corridor to Chabodoo scenic spot, Xinhua Lake scenic spot, Machieva Snow Mountain glaciers, mountain lakes and meadows, song and dance "A shame scraping" and so on. According to Weixi County Bureau of Statistics, the tourism industry in Weixi County has developed steadily during the 12th Five-Year Plan period. By 2015, the growth rate of 
tourism has increased.

Accessibility is the bottleneck restricting tourism development in Weixi County. Judging from the grade of the highway, the highways in Weixi County have basically achieved graded roads, but some of the major arteries are still outside roads, Mao Road. From a traffic perspective, from the time that other popular tourist destinations (Shangri-La, Lijiang, etc.) arrive in Weixi County, it takes 4-5 hours because of geographical environment and road traffic constraints. At present, the travel agency currently only launches a more mature tourism route, namely the 4th Meili Snow Mountain Tour (Lijiang-Shangri-La-Deqin-Weixi-Lijiang) in cooperation with the Off-Road Vehicle Club in Lijiang, and basically receives a tour every day. The team and others are traveling by car. The unity of the route and the road and traffic conditions greatly restrict the accessibility of tourists. The accessibility mentioned here mainly emphasizes the objective level, that is, traffic or communication accessibility. Specifically, commuting distances between Weixi County, Shangri-La City, and popular tourist destinations such as Lijiang City are still relatively far away due to constraints of terrain and development. Usually, it takes more than 3 hours to travel from Area A to Area B. There will be a sense of boredom, so if you can't shorten your commuting time, you'll have a negative psychological level of accessibility. At the same time, under the existing conditions, there is still a lack of traffic freedom. The constraints of accessibility make the current Weixi County insufficient to effectively attract large numbers of tourists.

Insufficient investment in tourism. There are altogether 2 tourism agencies in the county. By 2014, there were about 4,800 hospital beds. In the past 19 years, nearly 70 million yuan have been invested in tourism development. Accumulated so far, the government's investment in the development of tourism is not enough to build a large-scale scenic spot in Weixi County. This may be due to the state's emphasis on the development of tourism in Weixi County. The most important tourist destination in Diqing is Shangri-La City, and Deqin County in the other county also has a certain scale of development. The tourism development in Weixi County is always in the initial stage, and its development speed is far slower than the other two places. The second problem is the operation of tourism resources in Weixi County. Due to capital and operation and management reasons, currently the only tourist destination in Weixi County is the Shangri-La Golden Monkey National Park. Most of the other scenic spots are in a state of stagnation or unmanned management. The impact on the capital investment in tourism, and in the absence of funds running, the existing situation will not be improved, a cyclical cycle, resulting in the slow development of tourism in Weixi County.

Different tourism destinations exist in the image of tourist destinations, which are dominated by competition. It is also possible to use "image obscuration" as a representation. Image obstruction mainly refers to a number of tourist destinations distributed within a certain area. Among them, a tourist destination with a high level of tourism resources, distinctive tourism characteristics, and a product brand effect or a relatively strong market competitiveness, is The image will be more prominent, and the image of the surrounding other tourism sites will have a shadowing effect. [16] The Shangri-La neighbourhood around Weixi County is a typical tourist destination with relatively strong market competitiveness, which in turn leads to a clear "image masking" of Weixi County.

Although within the Diqing Prefecture, the tourism resources of one city and two counties have their own characteristics, especially ethnic cultural tourism resources. Weixi County, as the only Lisu Autonomous County in China, has obvious differences from other counties and cities. It is also very strong in the local area, so there is less shadowing effect caused by similar tourism resources. However, compared with other counties and cities, Weixi County lags behind due to various restrictive factors, tourism development is not enough, tourism product image is blurred, and overall tourism resources are lower than surrounding tourist destinations. This has led to Other tourist destinations in the surrounding areas will form "images".

In addition, in the Weixi County Tourism Development Plan, there is still the problem that the development vision is too ideal. In 2016, the Weixi County Tourism Bureau put forward the hopes of targeting the best hiking destinations in China and constructing the Sanjiang Parallel Flowing Visitors Center, and proposed the realization of a one-day tour of the land and the air. The Sanjiang 
tour route is near Weixi County. A few years to build goals. By integrating existing tourism resources in the county, they believe that Weixi County can create the best hiking tourism destination in China. However, in the author's field visits, access to relevant economic data, and questionnaire surveys, it can be concluded that Weixi County can be a transit destination from the perspective of existing tourism resources and various conditions, but it is difficult to become a tourist destination. . It can be said that at present, the most prominent problem in the tourism development in Weixi County is how to balance the constraints of the actual conditions, and it can basically meet the local vision for developing tourism and revitalizing the economy.

\section{Research on Tourism Development Path}

The definition of the word image in Western tourism studies is an abstract concept that contains past impressions, reputations, and evaluations among colleagues. The image also contains the expectations of users. [17] With the advent of the image age, the image has become a topic of widespread concern. The core idea of image orientation is: to manipulate what is already in the heart and re-integrate the existing linking relationship. [18] At present, the new normal of regional tourism development is in the same market competition area, and the regional development of different tourism destinations is coordinated. Considering that Shangri-La City and Lijiang City, with higher levels of tourism resources and attractive attractions, have obvious market screening effects on their neighboring Weixi County, how to form a spatially competitive relationship under existing conditions becomes Weixi. The important research factors of county tourism development. The synergy-based “image overlay" effect has become the key to promoting Weixi County tourism, attracting tourists, and activating the tourism development in Weixi County.

The tourism image of tourist destinations involves many aspects. It is a multi-level, multi-structured, complex system composed of many elements. The most important aspect of Weixi County's tourism image is to grasp its salient features that can be recognized, for example, its cognition of geographic location and geographical landscape, and the formation of a geographical spatial illustration. It can be said that any information on tourist attractions in Weixi County, including pictures, words, and speech, can be regarded as cognitive objects, and they can all be perceived by tourists and further influence them as a tourist destination in Weixi County. Image. The search for the distinctive feature of Weixi County is the positioning of tourism image in Weixi County.

In order to get out of "image obscurity" and use the "image superposition" effect, we must pay attention to forming a differentiated image orientation under the theme image. The Weixi County, which belongs to the Greater Shangri-La region, needs to differentiate itself from the tourism image of regions with higher levels of resources or higher level of tourism development, and has its own image characteristics. For example, it is the only She Autonomous County in the country and highlights its richness. The resources of the Lisu nationality intangible cultural heritage, such as the "Aquma scraping", which are ethnic characteristics of the Lisu nationality, can completely avoid the appearance of similarities with the surrounding tourist destinations. Furthermore, there is a more practical link between image overlays and travel route products. At present, Weixi County relies on Shangri-La's Golden Monkey National Park to form the only Lijiang-Shangri-La-Deqin-Weixi-Lijiang tourism route. Tourists often experience tourists due to geographical location and single tourist routes. Invisibility and little impression of Weixi County itself will not benefit the development of tourism in Weixi County in the long run. The differentiated image positioning proposal can cultivate Weixi County's own branded products and increase its comprehensive competitiveness. In this way, it can rely on different tourism products and combine different tourist routes with different tourism destinations. Thus pushing to different markets.

The two dimensions of time and space are the basic framework for the recognition of social, cultural, political and economic activities, and tourism activities are no exception. [19] In traditional studies, more consideration is given to the spatial agglomeration of tourism industry, but the spatial scalability of tourism flow should be taken into account. With the gradual extension of the upstream 
and downstream depths of the tourism industry chain, it provides the possibility of spatial overflow of tourism development. Spillover is the result of an externality of the behavioral subject's activity. Specifically, in an open economic system, the growth of the regional economy is greatly affected by the surrounding areas, and it shows the external characteristics of economic activity. According to the theory of new economic geography, the spillover regional process has ample spatial performance. [20] Tourism development also has a spatial spillover effect. Relevant evidence shows that tourism development in a certain area has a significant role in promoting economic growth in its neighboring regions. [21] How to form a spatial overflow of tourism development on economic growth? Perhaps it may be that the spatial concentration of the tourism industry and the spatial diffusion of the tourism flow have formed an interrelated effect, which is the result of the interaction between tourism supply and demand.

Taking Weixi County Tourism as an example, it can be said that the spatial diffusion of tourism flows is an important manifestation of tourism development. Although there are obvious spatial and temporal differences between the tourism economic development of Shangri-La and Weixi County, it is also very likely that Weixi County will have a diversion of tourist flows from Shangri-La and this possibility will, to a great extent, have a new impact on the economic growth of the region. Opportunity. Some scholars have discovered that the flow of tourists from tourist destinations in the core areas of the Shangri-La eco-tourism area of Shangri-La and Lijiang in the surrounding areas of Weixi County is clockwise. [22] That is to say, the current flow of tourists in the region is characterized by a back-and-forth reentry, because there is a lack of docking between each other, which also led to the difficulty of "little power" in Weixi County. On the other hand, if we can seek the means and links of docking in practice, we can divert tourists and increase the cost-effectiveness of regional tourism without increasing the cost of travel for tourists. This will further enhance Weixi County as a tourist destination. Charisma.

In general, the study of the image of tourism destinations will generally involve competition and cooperation between regions. As far as Weixi County is concerned, compared with other regions that are close to each other and connected to the line, especially in relation to other regions, the current conditions for tourism development in Weixi County are relatively lagging behind. The problem of realistic research value. From a realistic point of view, to avoid competitive disadvantages, we must further discuss the non-zero-sum game between regions. Some studies have shown that the best game between regions is to implement a "non-zero cooperation game", market-oriented, to break their respective regional barriers, find the best interests of their respective tourism development integration point, the implementation of regional strategic alliances. [twenty three]

The distinction between zero-sum games and non-zero-sum games is generally based on whether or not the sum of payoffs in the whole board is zero. The cooperation game can be regarded as a "win-win" strategy, so that we can obtain higher benefits. [24] Competition is the law of the market economy, and competition between tourism destinations is unavoidable, but how to use means or strategies in competition is varied. Weixi County can seek partnerships with surrounding tourism destinations so that it can jointly share the tourism market share. To a certain extent, Weixi County can quickly enhance the competitiveness of Weixi County in the tourism market.

"Shangri-La" has become a brand with strong influence and appeal because of its extensive familiarity around the world. The Shangri-La route has also been promoted overseas as one of China's twelve gold tourism routes and has become part of the country's image. The Shangri-La Ecotourism District is a broad geographical concept. In terms of administrative divisions, it includes the city of Jiuchuan and the Nine-Gegolia City, and Diqing Prefecture, which belongs to Weixi County, is within its core scope. In the surrounding areas of Weixi County, such as Shangri-La City, relying on the core scope of the Greater Shangri-La Ecosphere, the "Greater Shangri-La Tour and Distribution Center" is proposed. The Lijiang City, which is also close to Weixi County, has also played "Building Shangri-La Ecological. The gateway to the tourism circle and the central city." Shangri-La City and Lijiang City both belong to the earlier tourism development start-up tourism, and its tourism development is already at a mature stage. 
In contrast, the tourism industry in Weixi County is still in the initial stage. If its image orientation completely overlaps with them, it is obviously lacking in competitiveness and is not conducive to the future tourism development in Weixi County. Therefore, the strategy of "differentiating the image orientation under the theme image" mentioned in the image orientation of the tourism destination mentioned above will be adopted, and the concept of "harmony and difference" will be upheld. Relying on the backdrop of the large Shangri-La eco-tourism area, it strives to become a complementary tourism destination around popular tourist destinations. Deeply dig the characteristics of tourism resources and cultural resources in the county, emphasize "cultural self-confidence", enhance regional cultural soft power, use its own characteristics as a tourism destination for development resources, seek deeper cooperation with surrounding areas, and promote the realization of barrier-free travel within the region.

\section{Conclusion}

To sum up, the tourism development in Weixi County is still in the initial stage, and its image orientation should follow the strategy of "differentiating the image orientation under the theme image”, highlighting its rich ethnic cultural characteristics of the Lisu ethnic group and avoiding the surrounding tourist destinations. The image is similar. Through the use of the spatial diffusion of tourism flows, in actual practice, it seeks the channels and links for docking, diverts the tourism flow from the surrounding tourist destinations, and helps the tourism development in Weixi County. At the same time, they attempted to break down their respective regional barriers and introduced the concept of "non-zero and cooperative games" to seek partnerships with surrounding tourism destinations in order to enhance the competitiveness of Weixi County in the tourism market. Relying on the backdrop of the large Shangri-la eco-tourism area, it strives to become a complementary tourism destination around popular tourist destinations, collaborate with the development of regional tourism circles, and promote the realization of barrier-free travel within the region.

\section{Acknowledgements}

Fund Project: Ph.D. Program of Innovative Research Projects of Southwest Minzu University (CX2016BS02).

\section{References}

[1][7][16] Yang Zhenzhi, Chen Jin. Theory and Empirical Research on "Image Masking" and "Image Overlaying"[J]. Tourism Journal, 2003(3):62.

[2] Wang Lei, Liu Hongtao, Zhao Xiping. Research on the Connotation of Tourism Destination Image[J]. Journal of Xi'an Jiaotong University (Social Sciences), 1999(3):25,27.

[3] Li Leilei. Discussion on Urban Tourism Image Design [J]. Tourism Journal, 1998(1): 49.

[4] Wang Qianwen. A Review of Domestic Tourism Image Research[J].Yunnan Geographic Environment Research,2008(7):124.

[5] Wang Yanyong. Theory and Practice of Regional Tourism Development Strategy [J]. Economic Geography, 1999(1).

[6] Li Guoping, Ye Wen. A Preliminary Study on the Tourist Image Planning of Tourists Perceiving "Grayscale Districts"__An Image of the Tourism Image of Qujing City[J].Human Geography, 2002(4):35.

[8] Liu Ruiwen.A Study on the Asymmetric Function of Tourism Image[J].Geography and Geo-Information Science, 2007(4):75.

[9] Ma Xiaojing. Tourist Watching and Tourism Image Creation[J]. Tourism Journal, 2006(1):90-91. 
[10] Zhu Xiangbo.Analysis of the Image of Tourist Attractions in Panzhihua City Based on the Stereotype Angle[J].Urban Tourism Planning,2016(3):119.

[11] Wu Bihu.Yu Qing: A Review of the Research on the Development of Chinese Ethnic Cultural Tourism[J]. Ethnic Studies, 2000(4):88-89.

[12] Zhang Guohong. Chinese Cultural Tourism: Theory, Strategy and Practice [M]. Tianjin: Nankai University Press, 2001).

[13] LIU Xiaoying, WU Yusheng: Tourism Development and Ecological Construction and Urbanization Promotion in Ethnic Areas of Sichuan[J].Journal of Southwest University for Nationalities,2004(3):54.

[14] ZHANG Ji, XU Hongjian. Conflicts between subject and object role of family space in ethnic tourism regions: A case study of Baisha Village in Lijiang[J]. Geographical Sciences, 2016(7):1062.

[15] Weixi Lisu Autonomous County People's Government Development Research Center. Weixi Lisu Autonomous County Yearbook 2013-2014[J]. Kunming: Yunnan Ethnic Publishing House, 2015(9):32.

[17] HUANG Zhenfang, LI Xiang. Cognition and Promotion Model of Tourism Destination Image[J]. Tourism Journal, 2002, 17(3): 65-70.

[18] [United States] Ai Lisi, Jack Trout, Wang Enyi, Yu Shaowei, Translation. Positioning [M]. Beijing: China Financial and Economic Publishing House, 2002.

[19] Hall C. Time, space, tourism and social physics[J]. Tourism Recreation Research, 2005(1):93-98.

[20] CAO Honghua,WANG Rongcheng,LI Zheng.Study on Spatial Spillover Effects of Tourism Economics in Single-nuclear Tourism Circle_—A Case Study of Kunming-Northwest Tourism Circle[J].Resources Development \& Market,2013,29(12): 1319.

[21] ZHAO Lei, FANG Cheng, Wu Xiangming. Tourism Development, Spatial Spillover and Economic Growth: Empirical Evidence from China [J]. Tourism Journal, 2014(5):23.

[22] Yang Xiaoming. Research on the Cooperative Relationship of Tourism Development in Greater Shangri-la[J]. Areal Research and Development, 2013(6):73.

[23] WANG Yunlong. Game Analysis of Joint Promotion of Tourism Destination Images[J]. Journal of Shanghai Normal University (Philosophy and Social Sciences Edition), 2004(1):25.

[24] Jian Zhaoquan, Li Zheng. Formation Mechanism of Strategic Alliance: Non-zero-sum cooperation game[J]. Science of Science and Management of S. \& T. 1998(9):17. 